\title{
Observer-Based Robust Tracking Control for a Class of Switched Nonlinear Cascade Systems
}

\author{
Ben Niu, ${ }^{1}$ Deqing Zhao, ${ }^{2}$ Xudong Zhao, ${ }^{3}$ Hongyi Li, ${ }^{3}$ Xiangyong Chen, ${ }^{4}$ and Xiaoxiao Dong \\ ${ }^{1}$ College of Mathematics and Physics, Bohai University, Jinzhou 121001, China \\ 2 Jinzhou Heavy Water Pump Co., Ltd., Jinzhou 121001, China \\ ${ }^{3}$ College of Information Science and Technology, Bohai University, Jinzhou 121001, China \\ ${ }^{4}$ School of Scieces, Linyi University, Linyi 276005, China \\ ${ }^{5}$ College of Science, Shenyang University of Industry, Shenyang 110001, China
}

Correspondence should be addressed to Ben Niu; niubengj@163.com

Received 29 June 2013; Accepted 30 July 2013

Academic Editor: Rongni Yang

Copyright (c) 2013 Ben Niu et al. This is an open access article distributed under the Creative Commons Attribution License, which permits unrestricted use, distribution, and reproduction in any medium, provided the original work is properly cited.

This paper is devoted to robust output feedback tracking control design for a class of switched nonlinear cascade systems. The main goal is to ensure the global input-to-state stable (ISS) property of the tracking error nonlinear dynamics with respect to the unknown structural system uncertainties and external disturbances. First, a nonlinear observer is constructed through state transformation to reconstruct the unavailable states, where only one parameter should be determined. Then, by virtue of the nonlinear sliding mode control (SMC), a discontinuous nonlinear output feedback controller is designed using a backstepping like design procedure to ensure the ISS property. Finally, an example is provided to show the effectiveness of the proposed approach.

\section{Introduction}

Switched systems are a special class of hybrid systems in engineering applications and have attracted much attention from many researchers [1-8]. A switched system consists of a family of distinct active subsystems subject to a certain switching rule which chooses one of them being active during a certain time. The research on switched systems is motivated by two important practical considerations: (i) many realworld systems exhibit a fundamental characteristic of switching between different system structures; (ii) multicontroller switching provides an effective mechanism to handle highly complex systems and/or systems with large uncertainties. Therefore, switched systems have been a very active area of research in the past twenty years and have motivated a large and growing body of research work on a diverse array of issues, including modeling [9, 10], optimization [11, 12], stability analysis [13-17], and $H_{\infty}$ control [18-20].

Output feedback tracking control is a fundamentally important issue in control field and has been extensively studied over the last several decades. In the literature, several approaches have been developed to handle the output feedback control in the presence of structured or unstructured uncertainties: variable structure control approach [21], adaptive control approach [22], output dynamics controller with almost disturbance decoupling [23], and so forth. Inspired by these facts, for switched systems, output feedback tracking control is also a challenging issue for both theoretical investigation as well as practical applications [24-26]. Such a problem usually involves observer design [27], controller design [28], and switching law design [29]. However, to the best of the authors' knowledge, the output feedback tracking control of switched nonlinear cascade systems by designing nonlinear state observer has not been investigated yet.

In sliding mode control (SMC), sliding mode surface design and discontinuous reaching control law are two of the basic control issues. A common practice in SMC is to design a sliding mode surface according to the null space dynamics, which must ensure a stable sliding manifold when the system is in the sliding mode [30]. However, if there 
exist uncertainties in the null space nonlinear dynamics, sliding mode surface design becomes extremely difficult. Traditionally, the reaching control law is to force the system to reach and stay on the sliding mode surface. Nevertheless, this feature alone is no longer sufficient in the presence of unmatched uncertainties. Due to the effect of the unmatched uncertainties, the nonlinear dynamics may become divergent in a period shorter than the reaching time, if the input-tostate stable (ISS) property does not hold during the reaching phase. Hence, ISS property should be guaranteed either in the sliding phase or in the reaching phase.

In this paper, a class of switched nonlinear cascade systems with null space dynamics and range space dynamics are addressed for the tracking control task. Assuming that the full states are not available for measurement, the main objective of the paper is to ensure the global ISS property of the tracking error nonlinear dynamics while achieving a small tracking error bound. The features of the proposed approach are the following: (i) a nonlinear observer is designed for the switched system in which only one parameter needs to be determined; (ii) the resulting sliding manifold in the sliding phase possesses the desired ISS property and to certain extent the optimality through solving a Hamilton-Jacoby inequality; (iii) associated with the sliding mode surface, SMC is applied to the second subsystem that achieves the desired tracking.

Notations. We use standard notations throughout this paper. $\lambda_{\text {max }}(A)$ and $\lambda_{\text {min }}(A)$ stand for the maximum and minimum eigenvalues of a symmetric matrix $A$, respectively. $\{A\}_{\bar{n} \times \bar{n}}$ denotes the first $n$ rows and $n$ columns in $A$, and $\{A\}_{\underline{m} \times \underline{n}}$ denotes the last $m$ rows and $n$ columns in $A . R^{+}$denotes the set of nonnegative real numbers, $R^{n}$ denotes an $n$ dimension real vector space, $\|\cdot\|$ is the Euclidean norm and induced matrix norm, and $L_{\infty}[0, \infty)$ is the space of uniformly bounded functions on $[0, \infty) . D_{x} f=\partial f(x, y) / \partial x$ and $D_{y} f=\partial f(x, y) / \partial y$ are row vectors, and $\sigma(\cdot)$ denotes the largest singular value of a matrix.

\section{System Description and Problem Statement}

This paper is concerned with the following switched nonlinear cascade system described by

$$
\begin{aligned}
\dot{x}_{1}= & f_{1, \sigma(t)}\left(x_{1}, t\right)+B_{1, \sigma(t)}(t) x_{2}+H_{1, \sigma(t)}\left(x_{1}, t\right) \omega_{1}(t), \\
\dot{x}_{2}= & f_{2, \sigma(t)}(x, t)+B_{2, \sigma(t)}(t)\left[u_{\sigma(t)}+\Delta B_{2, \sigma(t)}(x, t)\right] \\
& \quad+H_{2, \sigma(t)}(x, t) \omega_{2}(t) \\
& y=
\end{aligned}
$$

where $x=\left[x_{1}^{T}, x_{2}^{T}\right]^{T}$ is a physically measurable state vector, $x_{1} \in R^{n}$ is the null space dynamics, $x_{2} \in R^{m}$ is the range space dynamics, and $\omega_{1} \in R^{h_{1}}$ and $\omega_{2} \in R^{h_{2}}$ are the external disturbance. $\sigma:[0, \infty) \rightarrow M=\{1,2, \ldots, m\}$ is the right continuous piecewise constant switching signal to be designed; $u_{i} \in R^{m}$ stands for the control input of the $i$ th subsystem, the mappings $f_{1, i}\left(x_{1}, t\right) \in R^{n}, f_{2, i}(x, t) \in$ $R^{m}, B_{1, i}(t) \in R^{n \times m}, B_{2, i}(t) \in R^{m \times m}, H_{1, i}\left(x_{1}, t\right) \in R^{n \times h_{1}}$, and
$H_{2, i}(x, t) \in R^{m \times h_{2}}$ are known and smooth with respect to $x$ and continuous with respect to time $t$, and $\Delta B_{2, i} \in R^{m}, i \in M$, denote the uncertainties in the control input. The relation $m \leq n$ holds for the system (1).

Corresponding to the switching signal $\sigma(t)$, we have the switching sequence

$$
\begin{gathered}
\sum=\left\{\left(x_{1}^{T}\left(t_{0}\right), x_{2}^{T}\left(t_{0}\right)\right)^{T} ;\left(i_{0}, t_{0}\right),\left(i_{1}, t_{1}\right), \ldots,\left(i_{k}, t_{k}\right), \ldots,\right. \\
\left.\mid i_{k} \in M, k=0,1, \ldots\right\},
\end{gathered}
$$

which means that the $i_{k}$ th subsystems are active when $t \in$ $\left[t_{k}, t_{k+1}\right)$. In addition, we assume that the state of the system (1) does not jump at the switching instants; that is, the trajectory $x(t)$ is everywhere continuous.

In this paper, the following assumptions are adopted to develop the main results.

Assumption 1. There exist two positive constants $b_{1}$ and $b_{2}$ such that for all $x_{1} \in R^{n}, t \geq 0$,

$$
0<b_{1}^{2} I_{m} \leq B_{1, i}^{T}(t) B_{1, i}(t) \leq b_{2}^{2} I_{m}, \quad i \in M,
$$

where $I_{m}$ is the identity matrix. Moreover, $B_{2, i}(t), i \in M$ are assumed to be invertible.

Assumption 2. The uncertainties $\omega_{1}(t), \omega_{2}(t)$ and $\eta_{i}(x, t), i \in$ $M$ in (1) are bounded as

$$
\begin{array}{r}
\left|\omega_{1}(t)\right| \leq l_{1}, \quad\left|\omega_{2}(t)\right| \leq l_{2}, \quad\left|\Delta B_{2, i}(x, t)\right| \leq l_{b}, \\
i \in M,
\end{array}
$$

where $l_{1}, l_{2}$, and $l_{b}$ are known positive constants.

In this paper, the output of the system (1) is required to track a given reference model: $y \Rightarrow y_{d}=x_{1 r}$; that is, the $x_{1}$ subpart is required to track the desired reference model

$$
\dot{x}_{1 r}=f_{r}\left(x_{1 r}, r(t), t\right)
$$

where $r(t)$ is a smooth reference input. Define the tracking error as $z_{1}=x_{1}-x_{1 r}$. Then, the error dynamics of the $x_{1}$ subpart can be transformed into

$$
\begin{aligned}
\dot{z}_{1}= & f_{1, i}\left(x_{1}, t\right)+B_{1, i}(t) x_{2}+H_{1, i}\left(x_{1}, t\right) \omega_{1}(t) \\
& -f_{r}\left(x_{1 r}, r(t), t\right), \quad i \in M .
\end{aligned}
$$

Assumption 3. There exists a smooth function $\psi(\cdot)$ such that the following matching condition holds:

$$
\begin{aligned}
f_{1, i} & \left(x_{1}, t\right)-f_{r}\left(x_{1 r}, r(t), t\right) \\
& =g_{1, i}\left(z_{1}, t\right)+B_{1, i}(t) \psi\left(x_{1}, x_{1 r}, r(t), t\right), \quad i \in M,
\end{aligned}
$$

where $\dot{\xi}=g_{1, i}(\xi, t)$ is asymptotically stable. 
According to Assumption 3, the error dynamics (6) and system (1) with the tracking objective (5) can be rewritten as

$$
\begin{aligned}
\dot{z}_{1}= & g_{1, i}\left(z_{1}, t\right)+B_{1, i}(t)\left[x_{2}+\psi\left(x_{1}, x_{1 r}, r(t), t\right)\right] \\
& +H_{1, i}\left(x_{1}, t\right) \omega_{1}(t), \\
\dot{x}_{2}= & f_{2, i}(x, t)+B_{2, i}(t)\left[u_{i}+\Delta B_{2, i}(x, t)\right] \\
& +H_{2, i}(x, t) \omega_{2}(t), \quad i \in M, \\
& \\
y= & x_{1} .
\end{aligned}
$$

Definition 4 (input-to-state stable (ISS) $[31,32]$ ). Consider a nonlinear dynamical system of the form

$$
\dot{x}=f(x, u),
$$

where $x$ and $u$ are the states and the inputs of (9), respectively. The system (9) is said to be locally input-to-state stable if there exist a class $K L$ function $\beta$, a class $K$ function $\gamma$, and constants $k_{1}, k_{2} \in R^{+}$such that

$$
\begin{array}{r}
\|x(t)\| \leq \beta\left(\left\|x_{0}\right\|, t\right)+\gamma\left(\left\|u_{T}(\cdot)\right\|_{L_{\infty}}\right), \\
\forall t \geq 0, \quad 0 \leq T \leq t,
\end{array}
$$

for all $x_{0} \in D$ and $u \in D_{u}$ satisfying $\left\|x_{0}\right\|<k_{1}$ and $\sup _{t>0}\left\|u_{T}(t)\right\|=\left\|u_{T}(\cdot)\right\|_{L_{\infty}}<k_{2}, 0 \leq T \leq t$. It is said to be input-to-state stable or globally ISS if $D=R^{n}, D_{u}=R^{m}$, and (10) is satisfied for any initial state and any bounded input $u$.

Control Objective. Under Assumptions 1-3, design a nonlinear observer for the system (1). Based on the observer, design a controller $u_{\sigma(t)}$ and a switching law $\sigma(t)$ such that

(i) the tracking error norm $\left\|z_{1}(t)\right\|$ in (8) tends to a ball $B_{s}$ in finite time, where the ball $B_{s}$ is defined as

$$
B_{s}=\left\{z_{1}(t):\left\|z_{1}(t)\right\| \leq s\right\}
$$

where $s$ is a positive constant;

(ii) the closed-loop system (8) possesses ISS property with respect to the disturbances $\omega_{i}=\left[\omega_{1}, \omega_{2}\right.$, $\left.\Delta B_{2, i}\right]^{T}, i \in M$.

\section{Nonlinear Observer Design}

This section is devoted to the design of a nonlinear observer for the system (1). Motivated by the work in [32, 33], a nonlinear observer is constructed through a state transformation which converts the system (1) into a new form such that the observer gain can be designed in a straightforward manner.

First, the system in (1) can be rewritten as the following:

$$
\begin{gathered}
\dot{x}=f_{i}(x, t)+k_{i}\left(u_{i}, t\right)+B_{i}(t) x+H_{i}(x, t) \omega_{i}(x, t), \\
y=C x, \quad i \in M,
\end{gathered}
$$

where $f_{i}(x, t)=\left[f_{1, i}\left(x_{1}, t\right), f_{2, i}(x, t)\right]^{T}$ and

$$
\begin{gathered}
k_{i}\left(u_{i}, t\right)=\left[\begin{array}{c}
0 \\
B_{2, i}(t) u
\end{array}\right], \quad B_{i}(t)=\left[\begin{array}{cc}
0 & B_{1, i}(t) \\
0 & 0
\end{array}\right], \\
\omega_{i}=\left[\begin{array}{c}
\omega_{1}(t) \\
\omega_{2}(t) \\
\Delta B_{2, i}(t)
\end{array}\right], \\
H_{i}(x, t)=\left[\begin{array}{ccc}
H_{1, i}\left(x_{1}, t\right) & 0 & 0 \\
0 & H_{2, i}(x, t) & B_{2, i}(t)
\end{array}\right], \\
C^{T}=\left[\begin{array}{c}
I_{n} \\
0_{m}
\end{array}\right] .
\end{gathered}
$$

Define the transformation matrices $T_{i}(t), i=1, \ldots, m$ and the matrices $\Xi_{\theta}, A$, and $\bar{C}$ as

$$
\begin{gathered}
T_{i}(t)_{2 n \times(n+m)}=\left[\begin{array}{cc}
I_{n} & 0 \\
0 & B_{1, i}(t)
\end{array}\right], \quad \Xi_{\theta}=\left[\begin{array}{cc}
I_{n} & 0 \\
0 & \frac{I_{n}}{\theta}
\end{array}\right], \\
A=\left[\begin{array}{cc}
0 & I_{n} \\
0_{n} & 0
\end{array}\right], \quad \bar{C}^{T}=\left[\begin{array}{l}
I_{n} \\
0_{n}
\end{array}\right] .
\end{gathered}
$$

Therefore, we obtain $\Xi_{\theta} A \Xi_{\theta}^{-1}=\theta A, \bar{C}^{T} \bar{C}_{\theta}=\bar{C}^{T} \bar{C}$, and

$$
\begin{gathered}
\zeta(t)=T_{i}(t) x=\left[\begin{array}{c}
x_{1} \\
B_{1, i}(t) x_{2}
\end{array}\right], \\
T_{i}(t) B_{i}(t)=A T_{i}(t) .
\end{gathered}
$$

Denote $T_{i}^{+}(t)$ as the left inverse of the matrix $T_{i}(t)$. Then, the $\zeta$ system can be written as

$$
\begin{aligned}
\dot{\zeta}= & T_{i}(t) \dot{x}+\dot{T}_{i}(t) x \\
= & T_{i}(t)\left[f_{i}(x, t)+k_{i}\left(u_{i}, t\right)\right. \\
+ & \left.B_{i}(t) x+H_{i}(x, t) \omega_{i}(x, t)\right]+\dot{T}_{i}(t) x \\
=A \zeta+T_{i} & {\left[f_{i}(x, t)+k_{i}\left(u_{i}, t\right)\right.} \\
& \left.+H_{i}(x, t) \omega_{i}(x, t)\right]+\dot{T}_{i} x \\
=A \zeta+T_{i} & {\left[f_{i}\left(T_{i}^{+} \zeta, t\right)+k_{i}\left(u_{i}, t\right)\right.} \\
& \left.+H_{i}\left(T_{i}^{+} \zeta, t\right) \omega_{i}\left(T_{i}^{+} \zeta, t\right)\right]+\dot{T}_{i}(t) T_{i}^{+} \zeta, \\
y=\bar{C} \zeta . \quad &
\end{aligned}
$$

Thus, the observer for the transformed $\zeta$ system in (16) can be constructed as

$$
\begin{aligned}
\dot{\hat{\zeta}}= & A \widehat{\zeta}+T_{i}\left[f_{i}\left(T_{i}^{+} \widehat{\zeta}, t\right)+k_{i}\left(u_{i}, t\right)\right] \\
& +\dot{T}_{i} T_{i}^{+} \widehat{\zeta}+\theta \Xi_{\theta}^{-1} P^{-1} \bar{C}^{T}(y-\bar{C} \widehat{\zeta})
\end{aligned}
$$

where $P$ is the symmetric positive definite solution of the following algebraic Lyapunov equation:

$$
P+A^{T} P+P A-\bar{C}^{T} \bar{C}=0 .
$$


Theorem 5. Assume that the system in (12) satisfies Assumptions 1-2. Then, under arbitrary switchings, the estimation error of the states has the following property:

$$
\left\|e_{x}(t)\right\|=\|x-\widehat{x}\| \leq q_{\theta}\left\|e_{x}(0)\right\|+\beta_{0} \varepsilon,
$$

where $q_{\theta}=\pi_{1} \theta \sqrt{\lambda_{\max }(P) / \lambda_{\min }(P)} e^{-\left(\left(\theta-c_{1}\right) / 2\right) t} \pi_{2}, \beta_{0}=$ $\pi_{1}\left(c_{2} \theta /\left(\theta-c_{1}\right) \sqrt{\lambda_{\min }(P)}\right), \varepsilon=\sqrt{l_{1}^{2}+l_{2}^{2}+l_{b}^{2}}$, and $c_{1}<\theta, c_{2}, \pi_{1}$, and $\pi_{2}$ are positive constants.

Proof. Define $e_{\zeta}(t)=\zeta(t)-\widehat{\zeta}(t)$. From (16) and (17), the estimation error dynamics of $e_{\zeta}(t)$ becomes

$$
\begin{aligned}
\dot{e}_{\zeta}= & \dot{\zeta}(t)-\dot{\bar{\zeta}}(t) \\
= & A \zeta+T_{i}\left[f_{i}\left(T_{i}^{+} \zeta, t\right)+k_{i}\left(u_{i}, t\right)\right. \\
& \left.\quad+H_{i}\left(T_{i}^{+} \zeta, t\right) \omega_{i}\left(T_{i}^{+} \zeta, t\right)\right]+\dot{T}_{i} T_{i}^{+} \zeta \\
& -A \widehat{\zeta}+T_{i}\left[f_{i}\left(T_{i}^{+} \widehat{\zeta}, t\right)+k_{i}\left(u_{i}, t\right)\right] \\
& +\dot{T}_{i} T_{i}^{+} \widehat{\zeta}+\theta \Xi_{\theta}^{-1} P^{-1} \bar{C}^{T}(y-\bar{C} \widehat{\zeta}) \\
= & \theta \Xi_{\theta}^{-1}\left(A-P^{-1} \bar{C}^{T} \bar{C}\right) \Xi_{\theta} e_{\zeta} \\
& +T_{i}\left[f_{i}\left(T_{i}^{+} \zeta, t\right)-f_{i}\left(T_{i}^{+} \widehat{\zeta}, t\right)\right]+\dot{T}_{i} T_{i}^{+} e_{\zeta} \\
& +T_{i} H_{i}\left(T_{i}^{+} \zeta, t\right) \omega_{i}\left(T_{i}^{+} \zeta, t\right) .
\end{aligned}
$$

Consider a transformation on the error as $\bar{e}_{\zeta}(t)=\Xi_{\theta} e_{\zeta}(t)$. Then, we have

$$
\begin{aligned}
\dot{\bar{e}}_{\zeta}= & \theta\left(A-P^{-1} \bar{C}^{T} \bar{C}\right) \bar{e}_{\zeta} \\
& +\Xi_{\theta} T_{i}\left[f_{i}\left(T_{i}^{+} \zeta, t\right)-f_{i}\left(T_{i}^{+} \widehat{\zeta}, t\right)\right] \\
& +\Xi_{\theta} \dot{T}_{i} T_{i}^{+} \Xi_{\theta}^{-1} \bar{e}_{\zeta}+\Xi_{\theta} T_{i} H_{i}\left(T_{i}^{+} \zeta, t\right) \omega_{i}\left(T_{i}^{+} \zeta, t\right) .
\end{aligned}
$$

Choosing $V_{1}=(1 / 2) \bar{e}_{\zeta} P \bar{e}_{\zeta}(t)$, where $P$ is the solution of (18), we obtain

$$
\begin{aligned}
\dot{V}_{1}= & -\theta V_{1}-\frac{\theta}{2} \bar{e}_{\zeta}^{T} \bar{C}^{T} \bar{C}_{\bar{e}} \\
& +\bar{e}_{\zeta}^{T} P \Xi_{\theta} T_{i}\left[f_{i}\left(T_{i}^{+} \zeta, t\right)-f_{i}\left(T_{i}^{+} \widehat{\zeta}, t\right)\right] \\
& +\bar{e}_{\zeta}^{T} P \Xi_{\theta} T_{i} H_{i}\left(T_{i}^{+} \zeta, t\right) \omega_{i}\left(T_{i}^{+} \zeta, t\right) \\
& +\bar{e}_{\zeta}^{T} P \Xi_{\theta} \dot{T}_{i} T_{i}^{+} \Xi_{\theta}^{-1} \bar{e}_{\zeta} \\
= & -\theta V_{1}+\bar{e}_{\zeta}^{T} P \Xi_{\theta} T_{i}\left[f_{i}\left(T_{i}^{+} \zeta, t\right)-f_{i}\left(T_{i}^{+} \widehat{\zeta}, t\right)\right] \\
& +\bar{e}_{\zeta}^{T} P \Xi_{\theta} T_{i} H_{i}\left(T_{i}^{+} \zeta, t\right) \omega_{i}\left(T_{i}^{+} \zeta, t\right) \\
& +\bar{e}_{\zeta}^{T} P \Xi_{\theta} \dot{T}_{i} T_{i}^{+} \Xi_{\theta}^{-1} \bar{e}_{\zeta} .
\end{aligned}
$$

For any $\theta>1$, we can infer that $\| \Xi_{\theta} T_{i}\left[f_{i}\left(T_{i}^{+} \zeta, t\right)-\right.$ $\left.f_{i}\left(T_{i}^{+} \widehat{\zeta}_{,}, t\right)\right]\left\|\leq b_{f}\right\| \bar{e}_{\zeta}\|,\| \Xi_{\theta} T_{i} H_{i}\left(T_{i}^{+} \zeta, t\right)\left\|\leq b_{h},\right\| \omega_{i}\left(T_{i}^{+} \zeta, t\right) \| \leq$ $\varepsilon$, and $\left\|\Xi_{\theta} \dot{T}_{i} T_{i}^{+} \Xi_{\theta}^{-1}\right\| \leq b_{t}$, where $b_{f}, b_{h}$, and $b_{t}$ do not depend on $\theta$. Then, (16) is transformed into

$$
\begin{aligned}
\dot{V}_{1} \leq & -\theta V_{1}+\lambda_{\max }(P)\left(b_{f}+b_{t}\right)\left\|\bar{e}_{\zeta}\right\|^{2} \\
& +\lambda_{\max }(P) b_{h} \varepsilon\left\|\bar{e}_{\zeta}\right\| \\
\leq & -\left(\theta-c_{1}\right) V_{1}+c_{2} \varepsilon \sqrt{V_{1}},
\end{aligned}
$$

where $c_{1}=2\left(\lambda_{\max }(P) / \lambda_{\min }(P)\right)\left(b_{f}+b_{t}\right)$ and $c_{2}=$ $\lambda_{\max }(P) b_{h}\left(\sqrt{2} / \sqrt{\lambda_{\min }(P)}\right)$. If $\theta>\max \left\{1+c_{1}\right\}$ is selected, then (23) becomes

$$
\begin{aligned}
\frac{d \sqrt{V_{1}}}{d t} \leq & -\frac{\left(\theta-c_{1}\right)}{2} \sqrt{V_{1}}+\frac{c_{2} \varepsilon}{2}, \\
\Longrightarrow \sqrt{V_{1}} \leq & e^{-\left(\left(\theta-c_{1}\right) / 2\right) t} \sqrt{V_{1}(0)} \\
& +\frac{c_{2} \varepsilon}{\theta-c_{1}}\left[1-e^{-\left(\left(\theta-c_{1}\right) / 2\right) t}\right], \\
\Longrightarrow\left\|\bar{e}_{\zeta}\right\| \leq & \sqrt{\frac{\lambda_{\max }(P)}{\lambda_{\min }(P)} e^{-\left(\left(\theta-c_{1}\right) / 2\right) t}\left\|\bar{e}_{\zeta}(0)\right\|} \\
& +\frac{c_{2} \varepsilon}{\left(\theta-c_{1}\right) \sqrt{\lambda_{\min }(P)}} .
\end{aligned}
$$

Using $\left\|\bar{e}_{\zeta}(t)\right\| \leq\left\|e_{\zeta}(t)\right\| \leq \theta\left\|\bar{e}_{\zeta}(t)\right\|$, (24) becomes

$$
\begin{aligned}
\left\|e_{\zeta}\right\| & \leq \theta \sqrt{\frac{\lambda_{\max }(P)}{\lambda_{\min }(P)}} e^{-\left(\left(\theta-c_{1}\right) / 2\right) t}\left\|\bar{e}_{\zeta}(0)\right\|+\frac{\theta c_{2} \varepsilon}{\left(\theta-c_{1}\right) \sqrt{\lambda_{\min }(P)}} \\
& \leq q_{\theta}^{1}\left\|e_{\zeta}(0)\right\|+\beta_{0}^{1} \varepsilon,
\end{aligned}
$$

where $q_{\theta}^{1}=\theta \sqrt{\lambda_{\max }(P) / \lambda_{\min }(P)} e^{-\left(\left(\theta-c_{1}\right) / 2\right) t}\left\|\bar{e}_{\zeta}(0)\right\|$ and $\beta_{0}^{1}=$ $\theta c_{2} /\left(\theta-c_{1}\right) \sqrt{\lambda_{\min }(P)}$. Thus, $\left\|e_{\zeta}(t)\right\|=\|\zeta(t)-\widehat{\zeta}(t)\| \leq$ $q_{\theta}^{1}\left\|e_{\zeta}(0)\right\|+\beta_{0}^{1} \varepsilon$. Furthermore, from Assumption 1, we have $\left\|T_{i}^{+}(t)\right\| \leq \pi_{1}$ and $\left\|T_{i}(t)\right\| \leq \pi_{2}$, where $\pi_{1}$ and $\pi_{2}$ are constants.

Based on $\zeta(t)=T(t) x(t)$ and $\widehat{\zeta}(t)=T(t) \widehat{x}(t)$, we get

$$
\begin{aligned}
\left\|e_{x}(t)\right\| & =\|x(t)-\widehat{x}(t)\|=\left\|T_{i}^{+} e_{\zeta}(t)\right\| \\
& \leq \pi_{1} q_{\theta}^{1} \pi_{2}\left\|e_{x}(0)\right\|+\pi_{1} \beta_{0}^{1} \chi=q_{\theta}\left\|e_{x}(0)\right\|+\beta_{0} \chi
\end{aligned}
$$

with $q_{\theta}=\pi_{1} q_{\theta}^{1} \pi_{2}, \beta_{0}=\pi_{1} \beta_{0}^{1}$. This completes the proof. 
From $(16)$ and $\dot{\bar{\zeta}}(t)=T_{i}(t) \dot{\vec{x}}(t)+\dot{T}_{i}(t) \hat{x}$, the observer to the original coordinate is

$$
\begin{aligned}
\dot{\hat{x}}(t)= & T_{i}^{+}(t)\left[\dot{\bar{\zeta}}(t)-\dot{T}_{i}(t) \hat{x}\right] \\
= & T_{i}^{+}(t)\left\{A \hat{\zeta}+T_{i}\left[f_{i}\left(T_{i}^{+} \hat{\zeta}, t\right)+k_{i}\left(u_{i}, t\right)\right]\right. \\
& +\dot{T}_{i} T_{i}^{+} \hat{\zeta}+\theta \Xi_{\theta}^{-1} P^{-1} \bar{C}^{T}(y-\bar{C} \hat{\zeta}) \\
& \left.-\dot{T}_{i}(t) \hat{x}\right\} \\
= & T_{i}^{+}(t) A T_{i} \hat{x}+f_{i}(\widehat{x}, t)+k_{i}\left(u_{i}, t\right) \\
& +\theta T_{i}^{+}(t) \Xi_{\theta}^{-1} P^{-1} \bar{C}^{T}(y-\bar{C} \hat{x}) \\
= & B_{i}(t) \hat{x}+f_{i}(\widehat{x}, t)+k_{i}\left(u_{i}, t\right) \\
& +\theta T_{i}^{+}(t) \Xi_{\theta}^{-1} P^{-1} \bar{C}^{T}(y-\bar{C} \hat{x}), \\
y= & x_{1} .
\end{aligned}
$$

Hence, the estimation error dynamics in the $x$-coordinate with $e_{x}(t)=x(t)-\widehat{x}(t)$ becomes

$$
\begin{aligned}
\dot{e}_{x}= & B_{i}(t) e_{x}+f_{i}(x, t)-f_{i}(\widehat{x}, t) \\
& +H_{i}(x, t) \omega_{i}(x, t)-\theta T_{i}^{+}(t) \Xi_{\theta}^{-1} P^{-1} \bar{C}^{T} \bar{C} e_{x} .
\end{aligned}
$$

\section{Controller Design and Stability Analysis}

Before the controller design, we would like to rewrite the observer dynamics in (27) as

$$
\begin{aligned}
& \dot{\hat{x}}_{1}=f_{1, i}\left(\widehat{x}_{1}, t\right)+B_{1, i}(t) \widehat{x}_{2}+\Phi_{n, i}, \\
& \dot{\hat{x}}_{2}=f_{2, i}(\widehat{x}, t)+B_{2, i}(t) u_{i}+\Phi_{m, i},
\end{aligned}
$$

where

$$
\begin{aligned}
\Phi_{n, i} & =\left\{\theta T_{i}^{+}(t) \Xi_{\theta}^{-1} P^{-1} \bar{C}^{T}\right\}_{\bar{n} \times \bar{n}}(y-C \hat{x}) \\
& =F_{n, i}(y-C \hat{x})=F_{n, i} \bar{C} e_{\zeta} \\
& =F_{n, i} \bar{C} \Xi_{\theta}^{-1} \bar{e}_{\zeta}=\bar{F}_{n, i} \bar{e}_{\zeta}, \\
\Phi_{m, i} & =\left\{\theta T_{i}^{+}(t) \Xi_{\theta}^{-1} P^{-1} \bar{C}^{T}\right\}_{m \times n}(y-C \widehat{x}) \\
& =F_{m, i}(y-C \hat{x})=F_{m, i} \bar{C} e_{\zeta} \\
& =F_{m, i} \bar{C} \Xi_{\theta}^{-1} \bar{e}_{\zeta}=\bar{F}_{m, i} \bar{e}_{\zeta} .
\end{aligned}
$$

Define $\widehat{z}_{1}=\widehat{x}_{1}-x_{1 r}$. In terms of the observer dynamics (22) and the desired trajectory (4), we have the following error dynamics:

$$
\begin{aligned}
\dot{\vec{z}}_{1}= & g_{1, i}\left(\widehat{z}_{1}, t\right) \\
& +B_{1, i}(t)\left[\widehat{x}_{2}+\psi\left(\widehat{x}_{1}, x_{1 r}, r(t), t\right)\right]+\Phi_{n, i}, \\
\dot{\hat{x}}_{2}= & f_{2, i}(\widehat{x}, t)+B_{2, i}(t) u_{i}+\Phi_{m, i} .
\end{aligned}
$$

In what follows, we first choose a sliding mode surface for the error dynamics of the null space dynamics $\widehat{z}_{1}$. Second, we design a controller for the augmented system in (21) and (32) such that ISS property is achieved.

Theorem 6. If there exist positive definite, radially unbounded, and smooth functions $V_{2, i}\left(\widehat{z}_{1}, t\right)$ and functions $\beta_{i j}\left(\widehat{z}_{1}, t\right) \leq$ $0, i, j=1, \ldots, m$ such that

$$
\begin{aligned}
& D_{t} V_{2, i}+\left(D_{\widehat{z}_{1}} V_{2, i}\right) g_{1} \\
& +\frac{1}{4 \gamma_{1}^{2}}\left(D_{\widehat{z}_{1}} V_{2, i}\right) \bar{F}_{n, i} \bar{F}_{n, i}^{T}\left(D_{\bar{z}_{1}} V_{2, i}\right)^{T} \\
& +\widehat{z}_{1}^{T} \widehat{z}_{1}+\sum_{j=1}^{m} \beta_{i j}\left(V_{2, j}-V_{2, i}\right) \leq 0,
\end{aligned}
$$

then, under the nonlinear sliding mode

$$
S=\widehat{x}_{2}+\psi=0
$$

and the switching law

$$
\sigma(t)=\min \left\{i \mid i=\max _{i \in M}\left\{V_{1, i}\left(e_{1}, t\right)\right\}\right\},
$$

the tracking error norm $\left\|z_{1}(t)\right\|$ tends to a ball $B_{s}$ in finite time, where the ball $B_{s}$ is defined as

$$
B_{s}=\left\{z_{1}(t):\left\|z_{1}(t)\right\| \leq \gamma_{3}^{2} \varepsilon^{2}=s\right\},
$$

where $\gamma_{3}$ and $\varepsilon$ are positive constants.

Proof. First, we now define the following piecewise Lyapunov function candidate:

$$
\begin{aligned}
\bar{V}_{2}\left(\bar{e}_{\zeta}, \bar{z}_{1}, t\right) & =V_{1}\left(\bar{e}_{\zeta}\right)+V_{2, \sigma}\left(\widehat{z}_{1}, t\right) \\
& =\frac{1}{2} \bar{e}_{\zeta} P \bar{e}_{\zeta}(t)+V_{2, \sigma}\left(\widehat{z}_{1}, t\right),
\end{aligned}
$$

where $V_{2, \sigma}\left(\widehat{z}_{1}, t\right)$ is switched among the solution $V_{2, i}\left(\widehat{z}_{1}, t\right)$ 's of (34) in accordance with the piecewise constant switching signal $\sigma$. 
Using the sliding mode surface constructed in (35) and under the switching law (36), the derivative of $V_{2, i}\left(\widehat{z}_{1}, t\right)$ is

$$
\begin{aligned}
\dot{V}_{2, i}= & D_{t} V_{2, i}+D_{\widehat{z}_{1}} V_{2, i} \\
& \times\left\{g_{1, i}+B_{1, i}(t)\right. \\
& \left.\times\left[\widehat{x}_{2}+\psi\left(\widehat{x}_{1}, x_{1 r}, r(t), t\right)\right]+\Phi_{n, i}\right\} \\
= & D_{t} V_{2, i}+D_{\widehat{z}_{1}} V_{2, i} g_{1, i}+D_{\widehat{z}_{1}} V_{2, i} \bar{F}_{n, i} \bar{e}_{\zeta} \\
= & D_{t} V_{i}+D_{e_{1}} V_{i} g_{1, i}+\gamma_{1}^{2} \bar{e}_{\zeta}^{T} \bar{e}_{\zeta} \\
& +\frac{1}{4 \gamma_{1}^{2}}\left(D_{\widehat{z}_{1}} V_{2, i}\right) \bar{F}_{n, i} \bar{F}_{n, i}^{T}\left(D_{\bar{z}_{1}} V_{2, i}\right)^{T} \\
& -\left\|\frac{1}{2 \gamma_{1}} \bar{F}_{n, i}^{T}\left(D_{\bar{z}_{1}} V_{2, i}\right)^{T}-\gamma_{1} \bar{e}_{\zeta}\right\|^{2} \\
\leq & D_{t} V_{i}+D_{e_{1}} V_{i} g_{1, i}+\gamma_{1}^{2} \omega_{1}^{2} \omega_{1} \\
& +\frac{1}{4 \gamma_{1}^{2}}\left(D_{\widehat{z}_{1}} V_{2, i}\right) \bar{F}_{n, i} \bar{F}_{n, i}^{T}\left(D_{\widehat{z}_{1}} V_{2, i}\right)^{T} .
\end{aligned}
$$

If there exist solutions of $V_{2, i}(\cdot)$ such that the inequality in (34) is satisfied, (39) becomes

$$
\dot{V}_{i} \leq-\widehat{z}_{1}^{T} \widehat{z}_{1}+\gamma_{1}^{2} \bar{e}_{\zeta}^{T} \bar{\zeta}_{\zeta} .
$$

From (22) and (40), we have

$$
\begin{aligned}
& \dot{\bar{V}}_{2}\left(\bar{e}_{\zeta}, \widehat{z}_{1}, t\right) \\
&=\dot{V}_{1}\left(\bar{e}_{\zeta}\right)+\dot{V}_{2, i}\left(\widehat{z}_{1}, t\right) \\
& \leq-\theta \bar{e}_{\zeta}^{T} P \bar{e}_{\zeta} \\
&+ \\
&+\bar{e}_{\zeta}^{T} P \Xi_{\theta} T_{i}\left[f_{i}\left(T_{i}^{+} \zeta, t\right)-f_{i}\left(T_{i}^{+} \bar{\zeta}, t\right)\right] \\
&+ \bar{e}_{\zeta}^{T} P \Xi_{\theta} T_{i} H_{i}\left(T_{i}^{+} \zeta, t\right) \omega_{i}\left(T_{i}^{+} \zeta, t\right) \\
&+ \bar{e}_{\zeta}^{T} P \Xi_{\theta} \dot{T}_{i} T_{i}^{+} \Xi_{\theta}^{-1} \bar{e}_{\zeta}-\widehat{z}_{1}^{T} \widehat{z}_{1}+\gamma_{1}^{2} e_{\zeta}^{T} \bar{e}_{\zeta} \\
& \leq-\theta \lambda_{\min }(P)\left\|\bar{e}_{\zeta}\right\|^{2} \\
&+\lambda_{\max }(P)\left(b_{f}+b_{t}\right)\left\|\bar{e}_{\zeta}\right\|^{2} \\
&+\bar{e}_{\zeta}^{T} P \Xi_{\theta} T_{i} H_{i}\left(T_{i}^{+} \zeta, t\right) \omega_{i}\left(T_{i}^{+} \zeta, t\right) \\
&-\widehat{z}_{1}^{T} \widehat{z}_{1}+\gamma_{1}^{2} \bar{e}_{\zeta}^{T} \bar{e}_{\zeta} \\
& \leq-\left[\theta \lambda_{\min }(P)\left\|\bar{e}_{\zeta}\right\|^{2}\right. \\
&-\gamma_{1}^{2}-\lambda_{\max }(P)\left(b_{f}+b_{t}\right) \\
&\left.-\frac{1}{4 \gamma_{2}^{2}} \lambda_{\max }(P)^{2} b_{h}^{2}\right]\left\|\bar{e}_{\zeta}\right\|^{2} \\
&- \widehat{z}_{1}^{T} \widehat{z}_{1}+\gamma_{2}^{2}\left\|\omega_{i}\left(T_{i}^{+} \zeta, t\right)\right\|^{2} .
\end{aligned}
$$

When $\theta$ is selected to be

$$
\begin{gathered}
\theta \lambda_{\min }(P)\left\|\bar{e}_{\zeta}\right\|^{2}-\gamma_{1}^{2}-\lambda_{\text {max }}(P)\left(b_{f}+b_{t}\right) \\
-\frac{1}{4 \gamma_{2}^{2}} \lambda_{\text {max }}(P)^{2} b_{h}^{2} \geq 0,
\end{gathered}
$$

then the inequality (41) becomes

$$
\dot{\bar{V}}_{2}\left(\bar{e}_{\zeta}, \widehat{z}_{1}, t\right) \leq-\widehat{z}_{1}^{T} \widehat{z}_{1}+\gamma_{2}^{2}\left\|\omega_{i}\left(T_{i}^{+} \zeta, t\right)\right\|^{2} .
$$

Using $e_{x, 1}(t)=x_{1}(t)-\widehat{x}_{1}(t)=z_{1}(t)-\widehat{z}_{1}(t),\left\|\widehat{z}_{1}(t)\right\|^{2} \geq$ $\left\|z_{1}(t)\right\|^{2}-\left\|e_{x, 1}(t)\right\|^{2}$, and $e_{x, 1}(t)=\bar{e}_{\zeta, 1}(t)$ and according to $e_{x}(t)=T_{i}^{+} \Xi_{\theta}^{-1} \bar{e}(t),(43)$ becomes

$$
\begin{aligned}
& \dot{\bar{V}}_{2}\left(\bar{e}_{\zeta}, \widehat{z}_{1}, t\right) \\
& \quad \leq-z_{1}^{T} z_{1}+\left\|e_{x, 1}(t)\right\|^{2}+\gamma_{2}^{2}\left\|\omega_{i}\left(T_{i}^{+} \zeta, t\right)\right\|^{2} \\
& \quad=-z_{1}^{T} z_{1}+\left\|e_{\zeta, 1}(t)\right\|^{2}+\gamma_{2}^{2}\left\|\omega_{i}\left(T_{i}^{+} \zeta, t\right)\right\|^{2} \\
& \quad \leq-z_{1}^{T} z_{1}+\left\|e_{\zeta}(t)\right\|^{2}+\gamma_{2}^{2}\left\|\omega_{i}\left(T_{i}^{+} \zeta, t\right)\right\|^{2} .
\end{aligned}
$$

From (17), $\bar{e}_{\zeta}$ is bounded as

$$
\left\|\bar{e}_{\zeta}\right\| \leq \frac{\lambda_{\max }(P) b_{h}}{\left[\lambda_{\min }(P) \theta-\lambda_{\max }(P)\left(b_{f}+b_{t}\right)\right]}\left\|\omega_{i}\left(T_{i}^{+} \zeta, t\right)\right\|,
$$

where $\lambda_{\min }(P) \theta-\lambda_{\max }(P)\left(b_{f}+b_{t}\right)>0$ according to (42). Hence, (44) becomes

$$
\begin{aligned}
\dot{\bar{V}}_{2}\left(\bar{e}_{\zeta}, \widehat{z}_{1}, t\right) & \leq-z_{1}^{T} z_{1}+\gamma_{3}^{2}\left\|\omega_{i}\left(T_{i}^{+} \zeta, t\right)\right\|^{2} \\
& \leq-z_{1}^{T} z_{1}+\gamma_{3}^{2} \varepsilon^{2},
\end{aligned}
$$

where $\gamma_{3}=\sqrt{\gamma_{2}^{2}+\chi^{2}}$ and $\chi=b_{h} /\left(\left(\lambda_{\min }(P) / \lambda_{\max }(P)\right)-\right.$ $\left.\lambda_{\max }(P)\left(b_{f}+b_{t}\right)\right)$. Note that $\lim _{\theta \rightarrow \infty} \chi=0$. Equation (47) shows that the tracking error norm $\left\|z_{1}(t)\right\|$ in (8) tends to a ball in finite time, which is defined by

$$
B_{s}=\left\{z_{1}(t):\left\|z_{1}(t)\right\| \leq \gamma_{3}^{2} \varepsilon^{2}=s\right\},
$$

where $B_{s}=\left\{z_{1}(t):\left\|z_{1}(t)\right\| \leq \gamma_{3}^{2} \varepsilon^{2}=s\right\}, \varepsilon=\sqrt{l_{1}^{2}+l_{2}^{2}+l_{\eta}^{2}}$.

Remark 7. In the nonlinear uncertain system (32), if $g_{1, i}\left(\widehat{z}_{1}, t\right)$ can be expressed as $W_{1, i}\left(\widehat{z}_{1}, t\right) \widehat{z}_{1}$, when $W_{1, i}\left(\widehat{z}_{1}, t\right)$ is a matrixvalued smooth function, then the HJI inequality (34) can be simplified into the following differential Riccati inequality:

$$
\begin{aligned}
\frac{1}{2} \dot{E}_{i} & +\frac{1}{2}\left(E_{i} W_{1, i}+W_{1, i}^{T} E_{i}\right)+\frac{1}{4 \gamma_{1}^{2}} E_{i} \bar{F}_{n, i} \bar{F}_{n, i}^{T} E_{i} \\
+ & I_{n \times n}+\sum_{j=1}^{m} \beta_{i j}\left(E_{j}-E_{i}\right) \leq 0,
\end{aligned}
$$

where $E_{i}\left(\widehat{z}_{1}, t\right) \widehat{z}_{1}, i \in M$, are symmetric positive definite smooth matrices. 
Remark 8. In the observer design, the parameter $\theta$ is the only key parameter to be determined. It should be designed such that the two conditions are satisfied in (42) and $\theta=\max \left\{1, c_{1}\right\}$ simultaneously.

Remark 9. Since the estimation error of the states in Theorem 5 has the property (19), the tracking error simply converges to a ball showed in (37).

We are now in a position to design the controller to ensure the ISS stability.

Theorem 10. With the sliding mode surface (35), the switching law (36), and the following sliding mode controller

$$
\begin{aligned}
& u_{i}= u_{1, i}+u_{2, i}, \\
& u_{1, i}=-B_{2, i}^{-1}[ D_{t} S+\left(D_{x_{1 r}} S\right) \dot{x}_{1 r} \\
&\left.\quad+L\left(f_{1, i}+B_{1, i} \widehat{x}_{2}+\Phi_{n, i}\right)+f_{2, i}+\Phi_{m, i}\right], \\
& \\
& u_{2, i}=-k_{0} \frac{B_{2, i}^{T} S}{\left\|B_{2, i}^{T} S\right\|},
\end{aligned}
$$

where $L\left(\widehat{x}_{1}, x_{1 r}, t\right)=D_{\widehat{x}_{1}} S \in R^{m \times n}$ and $k_{0}$ is a positive constant, the system (8) is globally ISS stable with respect to the external disturbance inputs, and the tracking error norm $\left\|z_{1}\right\|$ is bounded in $B_{s}$ as in Theorem 6.

Proof. Define $V_{3}=(1 / 2) S^{T} S$. Choose the following piecewise Lyapunov function candidate:

$$
\begin{aligned}
\bar{V}_{3}\left(\bar{e}_{\zeta}, \widehat{z}_{1}, \widehat{x}, x_{1 r}, t\right) & =V_{1}\left(\bar{e}_{\zeta}\right)+V_{2, \sigma}\left(\widehat{z}_{1}, t\right)+\frac{1}{2} S^{T} S \\
& =\frac{1}{2} \bar{e}_{\zeta} P \bar{e}_{\zeta}(t)+V_{2, \sigma}\left(\widehat{z}_{1}, t\right) \frac{1}{2} S^{T} S
\end{aligned}
$$

where $V_{2, \sigma}\left(\widehat{z}_{1}, t\right)$ is switched among the solution $V_{2, i}\left(\widehat{z}_{1}, t\right)$ 's of (34) in accordance with the piecewise constant switching signal $\sigma$.

Then, we have

$$
\begin{aligned}
\dot{V}_{3}= & S^{T}\left[D_{t} S+\left(D_{x_{1 r}} S\right) \dot{x}_{1 r}+L\left(f_{1, i}+B_{1, i} \widehat{x}_{2}+\Phi_{n, i}\right)\right. \\
& \left.+f_{2, i}+B_{2, i} u_{i}+\Phi_{m, i}\right] \\
\leq & -k_{0}\left\|B_{2, i}^{T} S\right\| .
\end{aligned}
$$

From (44) and (54), we have

$$
\begin{aligned}
\dot{\bar{V}}_{3} & =\dot{\bar{V}}_{2}+\dot{V} \leq-z_{1}^{T} z_{1}+\gamma_{3}^{2}\left\|\omega_{i}\left(T_{i}^{+} \zeta, t\right)\right\|^{2}-k_{0}\left\|B_{2, i}^{T} S\right\| \\
& \leq-z_{1}^{T} z_{1}+\gamma_{3}^{2} \varepsilon^{2},
\end{aligned}
$$

which implies that the system (8) is globally ISS with respect to the external disturbance input, and the tracking error norm $\left\|z_{1}\right\|$ is bounded in $B_{s}$ in finite time.

\section{Illustrative Example}

In this section, we present a simulation example to illustrate the applicability and effectiveness of the proposed approach.

Example 1. Consider a switched nonlinear cascade system as in (1), where

$$
\begin{aligned}
& f_{1,1}=f_{1,2}=W_{1} x_{1}=\left[\begin{array}{cc}
0 & 1 \\
-1.6 & -2.1
\end{array}\right]\left[\begin{array}{l}
x_{11} \\
x_{12}
\end{array}\right], \\
& B_{1,1}(t)=\left[\begin{array}{cc}
1+0.8 \sin (t) & 0 \\
0 & 1
\end{array}\right] \text {, } \\
& B_{1,2}(t)=\left[\begin{array}{cc}
1+0.8 \cos (t) & 0 \\
0 & 1
\end{array}\right] \text {, } \\
& H_{1,1}=\left[\begin{array}{ll}
\sin \left(x_{11}\right) & \cos \left(x_{11}\right) \\
\cos \left(x_{12}\right) & \sin \left(x_{12}\right)
\end{array}\right] \text {, } \\
& H_{1,2}=\left[\begin{array}{ll}
\cos \left(x_{12}\right) & \sin \left(x_{12}\right) \\
\sin \left(x_{11}\right) & \cos \left(x_{11}\right)
\end{array}\right] \text {, } \\
& \omega_{1}=\left[e^{-0.3 t}, e^{-0.1 t}\right]^{T}, \quad \omega_{2}=\left[e^{-0.2 t}, e^{-0.5 t}\right]^{T}, \\
& f_{2,1}=2 x_{11}^{2} x_{12} \cos \left(x_{2}\right) \text {, } \\
& f_{2,1}=x_{12} \sin \left(x_{12} x_{2}\right) \text {, } \\
& \eta_{1}=0.6 \sin \left(x_{11}\right)+0.6 \sin \left(x_{12}\right) \text {, } \\
& \eta_{2}=-0.6 \cos \left(x_{12}\right)-0.6 \cos \left(x_{2}\right) \text {. }
\end{aligned}
$$

The nonlinear observer is designed as in (27). Based on (18), we have the symmetric positive definite solution

$$
P=\left[\begin{array}{ccc}
1 & 0 & -1 \\
0 & 1 & 0 \\
-1 & 0 & 2
\end{array}\right] .
$$

Then, $\theta=86$ is selected based on Remark 8 with $b_{f}=$ $4.8416, b_{t}=1, b_{h}=1.128$, and $\gamma_{2}=1$.

The target trajectory is $x_{11 r}=0.3 \sin (\pi t)$ and $x_{12 r}=$ $\dot{x}_{11 r}=0.3 \pi \cos (\pi t)$. From (8), the error dynamics of the $x_{1}$ subpart can be expressed as

$$
\dot{z}_{1}=W_{1} e_{1}+B_{1 . i}\left[x_{2}+\psi\right]+H_{1, i} \omega_{1}, \quad i=1,2,
$$

where $\psi(t)=-\dot{x}_{12 r}-1.6 x_{11 r}-2.1 x_{12 r}$.

Let $\gamma_{1}=1$. In $\widehat{z}_{1}$-subpart, according to Remark 7, we first choose $V_{i}\left(\widehat{z}_{1}, t\right)=(1 / 2) \widehat{z}_{1}^{T} E_{i} \widehat{z}_{1}, i=1,2$, where $E_{i}$ are determined by the differential Riccati inequality (48). When $\dot{E}_{i}=0$ and $\beta_{12}=\beta_{21}=-1$, from the linear algebraic matrix inequality

$$
\begin{aligned}
& \frac{1}{2}\left(E_{1} W_{1}+W_{1}^{T} E_{1}\right)+\frac{1}{4 \gamma_{1}^{2}} E_{1} H_{1,1} H_{1,1}^{T} E_{1} \\
& \quad+I+\left(E_{2}-E_{1}\right) \leq 0, \\
& \frac{1}{2}\left(E_{2} W_{1}+W_{1}^{T} E_{2}\right)+\frac{1}{4 \gamma_{1}^{2}} E_{2} H_{1,2} H_{1,2}^{T} E_{2} \\
& \quad+I+\left(E_{1}-E_{2}\right) \leq 0,
\end{aligned}
$$




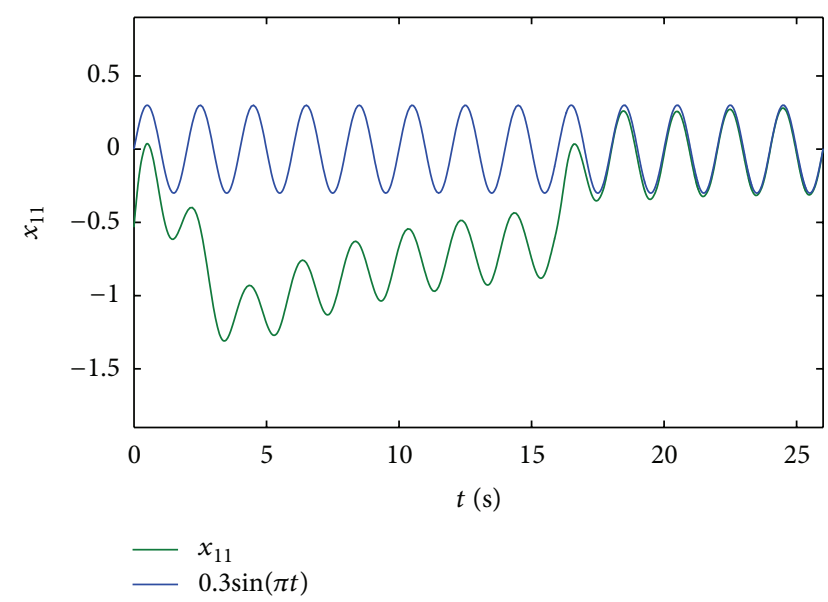

FIGURE 1: The response of the state $x_{11}$.

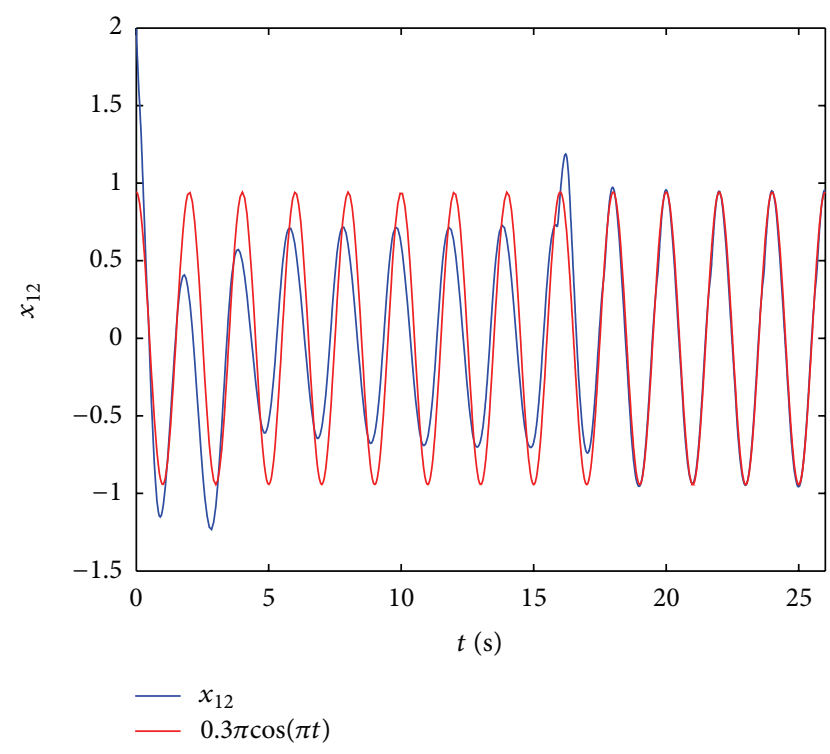

FIgURE 2: The response of the state $x_{12}$.

and using the singular values of the matrices $H_{1,1}$ and $H_{1,2}$, we can get two symmetric positive definite smooth matrices

$$
\begin{gathered}
E_{1}=\left[\begin{array}{ll}
0.114799 & 0.000330 \\
0.000330 & 0.463736
\end{array}\right], \\
E_{2}=\left[\begin{array}{cc}
0.039253 & -1.025477 \\
-1.025477 & 0.070260
\end{array}\right] .
\end{gathered}
$$

Therefore, the switching surface is $S=\widehat{x}_{2}+\psi=\widehat{x}_{2}-\dot{x}_{12 r}-$ $1.6 x_{11 r}-2.1 x_{12 r}$. Moreover, the switching law is chosen as

$$
\sigma(t)= \begin{cases}1 & \text { if } \widehat{z}_{1}^{T} E_{1} \widehat{z}_{1}>\widehat{z}_{1}^{T} E_{2} z_{1}, \\ 2 & \text { if } \widehat{z}_{1}^{T} E_{2} z_{1}>\widehat{z}_{1}^{T} E_{1} z_{1},\end{cases}
$$

according to (36) in Theorem 6, and the controller is constructed according to (49) in Theorem 10.

Let the initial states be $(-0.6,1.9,-0.56)^{T}$. Figures 1 and 2 show the responses of the states $x_{11}$ and $x_{12}$, respectively.

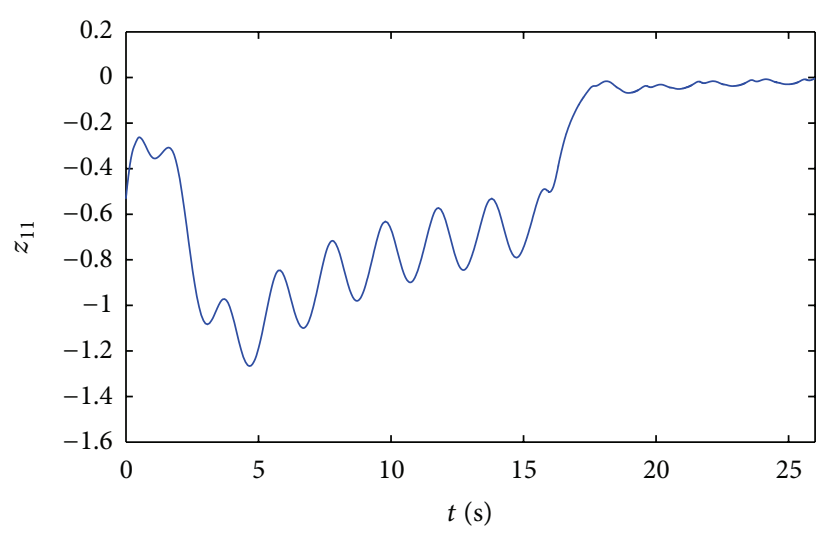

FIgURE 3: The evolution of the tracking error $z_{11}$.

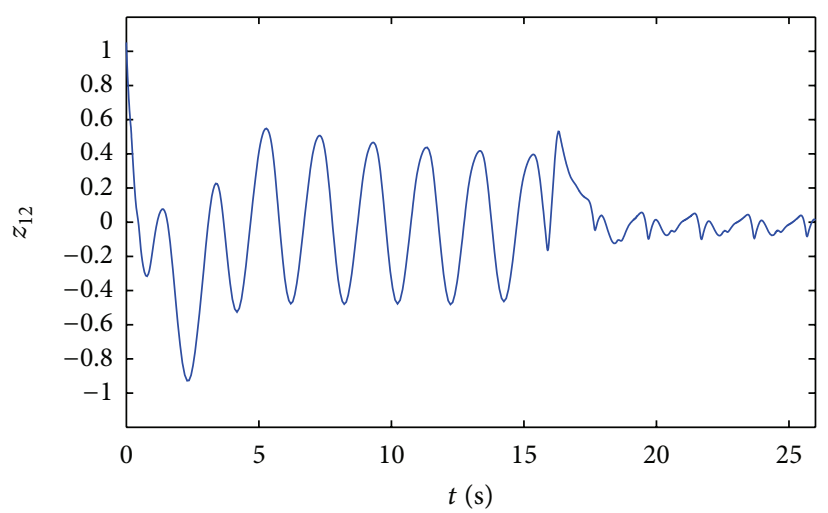

FIGURE 4: The evolution of the tracking error $z_{12}$.

The tracking errors $z_{11}$ and $z_{12}$ are shown in Figures 3 and 4 , respectively, which demonstrate the tracking errors of the states $x_{11}$ and $x_{12}$ that are bounded with fast convergence. All the figures indicate the feasibility of our results.

\section{Conclusions}

In this paper, we have investigated the tracking control problem for a class of switched nonlinear cascade systems with unknown system uncertainties and external disturbances. A new robust output feedback control approach based on a nonlinear observer is proposed for the switched system. Through solving a Hamilton-Jacoby inequality, the nonlinear control law for the first subsystem specifies a nonlinear sliding mode surface. By virtue of nonlinear control for the first subsystem, the resulting sliding manifold in the sliding phase possesses the desired ISS property. Furthermore, sufficient conditions for the solvability of the tracking control problem of the switched systems and design of both switching law and output feedback controller are presented.

\section{Acknowledgments}

This work was supported by the National Natural Science Foundation of China under Grants (nos. 61304054, 61203002, 
61304102, and 61203123), the Program for New Century Excellent Talents in University, the Program for Liaoning Excellent Talents in University, and the Project of Shandong Province Higher Educational Science and Technology Program under Grant (no. J13LI11).

\section{References}

[1] D. Liberzon and A. S. Morse, "Basic problems in stability and design of switched systems," IEEE Control Systems Magazine, vol. 19, no. 5, pp. 59-70, 1999.

[2] J. Lian, C. W. Mu, and P. Shi, "Asynchronous $H_{\infty}$ filtering for switched stochastic systems with time-varying delay," Information Sciences, vol. 224, no. 1, pp. 200-212, 2013.

[3] J. Xiong, J. Lam, H. Gao, and D. W. C. Ho, "On robust stabilization of Markovian jump systems with uncertain switching probabilities," Automatica, vol. 41, no. 5, pp. 897-903, 2005.

[4] P. Mhaskar, N. H. El-Farra, and P. D. Christofides, "Predictive control of switched nonlinear systems with scheduled mode transitions," IEEE Transactions on Automatic Control, vol. 50, no. 11, pp. 1670-1680, 2005.

[5] S. Yin, H. Luo, and S. Ding, "Real-time implementation of faulttolerant control systems with performance optimization," IEEE Transactions on Industrial Electronics, 2013.

[6] J. Daafouz, P. Riedinger, and C. Iung, "Stability analysis and control synthesis for switched systems: a switched Lyapunov function approach," IEEE Transactions on Automatic Control, vol. 47, no. 11, pp. 1883-1887, 2002.

[7] R. Wang, J. Zhao, G. M. Dimirovski, and G.-P. Liu, "Output feedback control for uncertain linear systems with faulty actuators based on a switching method," International Journal of Robust and Nonlinear Control, vol. 19, no. 12, pp. 1295-1312, 2009.

[8] L. Zhang, P. Shi, and M. Basin, "Robust stability and stabilisation of uncertain switched linear discrete time-delay systems," IET Control Theory and Applications, vol. 2, no. 7, pp. 606-614, 2008.

[9] Z. Shu, J. Lam, and J. Xiong, "Static output-feedback stabilization of discrete-time Markovian jump linear systems: a system augmentation approach," Automatica, vol. 46, no. 4, pp. 687694, 2010.

[10] C. K. Ahn, "Passive learning and input-to-state stability of switched Hopfield neural networks with time-delay," Information Sciences, vol. 180, no. 23, pp. 4582-4594, 2010.

[11] M. S. Branicky, "Multiple Lyapunov functions and other analysis tools for switched and hybrid systems," IEEE Transactions on Automatic Control, vol. 43, no. 4, pp. 475-482, 1998.

[12] S.-L. Dai, H. Lin, and S. S. Ge, "Scheduling-and-control codesign for a collection of networked control systems with uncertain delays," IEEE Transactions on Control Systems Technology, vol. 18, no. 1, pp. 66-78, 2010.

[13] J. Lian and K. Zhang, "Exponential stability for switched CohenGrossberg neural networks with average dwell time," Nonlinear Dynamics, vol. 63, no. 3, pp. 331-343, 2011.

[14] A. Ibeas and M. de la Sen, "Exponential stability of simultaneously triangularizable switched systems with explicit calculation of a common Lyapunov function," Applied Mathematics Letters, vol. 22, no. 10, pp. 1549-1555, 2009.

[15] H. Xu and K. L. Teo, "Robust stabilization of uncertain impulsive switched systems with delayed control," Computers and Mathematics with Applications, vol. 56, no. 1, pp. 63-70, 2008.
[16] K. Mathiyalagan, R. Sakthivel, and S. M. Anthoni, "New robust exponential stability results for discrete-time switched fuzzy neural networks with time delays," Computers \& Mathematics with Applications, vol. 64, no. 9, pp. 2926-2938, 2012.

[17] Z. Zhang and X. Liu, "Robust stability of uncertain discrete impulsive switching systems," Computers and Mathematics with Applications, vol. 58, no. 2, pp. 380-389, 2009.

[18] B. Niu and J. Zhao, "Stabilization and $L_{2}$-gain analysis for a class of cascade switched nonlinear systems: an average dwell-time method," Nonlinear Analysis: Hybrid Systems, vol. 5, no. 4, pp. 671-680, 2011.

[19] B. Niu and J. Zhao, "Robust $H_{\infty}$ control for a class of switched nonlinear cascade systems via multiple Lyapunov functions approach," Applied Mathematics and Computation, vol. 218, no. 11, pp. 6330-6339, 2012.

[20] M. S. Mahmoud, "Switched discrete-time systems with timevarying delays: a generalized $\mathrm{H}_{2}$ approach," Computers and Mathematics with Applications, vol. 57, no. 1, pp. 79-95, 2009.

[21] S. Oh and H. K. Khalil, "Nonlinear output-feedback tracking using high-gain observer and variable structure control," Automatica, vol. 33, no. 10, pp. 1845-1856, 1997.

[22] M. Jankovic, "Adaptive nonlinear output feedback tracking with a partial high-gain observer and backstepping," IEEE Transactions on Automatic Control, vol. 42, no. 1, pp. 106-113, 1997.

[23] R. Marino and P. Tomei, "Nonlinear output feedback tracking with almost disturbance decoupling," IEEE Transactions on Automatic Control, vol. 44, no. 1, pp. 18-28, 1999.

[24] L. Rodrigues and J. P. How, "Observer-based control of piecewise-affine systems," International Journal of Control, vol. 76, no. 5, pp. 459-477, 2003.

[25] Z. G. Li, C. Y. Wen, and Y. C. Soh, "Observer-based stabilization of switching linear systems," Automatica, vol. 39, no. 3, pp. 517524, 2003.

[26] D. Xie, N. Xu, and X. Chen, "Stabilisability and observerbased switched control design for switched linear systems," IET Control Theory and Applications, vol. 2, no. 3, pp. 192-199, 2008.

[27] A. Alessandri and P. Coletta, "Design of Luenberger observers for a class of hybrid linear systems," in Hybrid Systems: Computation and Control, Lecture Notes in Computer Science, pp. 7-18, 2001.

[28] A. L. Juloski, W. P. M. H. Heemels, and S. Weiland, "Observer design for a class of piecewise linear systems," International Journal of Robust and Nonlinear Control, vol. 17, no. 15, pp. 13871404, 2007.

[29] J. C. Geromel, P. Colaneri, and P. Bolzern, "Dynamic output feedback control of switched linear systems," IEEE Transactions on Automatic Control, vol. 53, no. 3, pp. 720-733, 2008.

[30] C. Edwards and S. K. Spurgeon, Sliding Mode Control: Theory and Applications, Taylor and Francis, London, UK, 1998.

[31] H. J. Marquez, Nonlinear Control Systems: Analysis and Design, Wiley-Interscience, 2003.

[32] K. K. Busawon and M. Saif, "A state observer for nonlinear systems," IEEE Transactions on Automatic Control, vol. 44, no. 11, pp. 2098-2103, 1999.

[33] M. Farza, M. M'Saad, and L. Rossignol, "Observer design for a class of MIMO nonlinear systems," Automatica, vol. 40, no. 1, pp. 135-143, 2004. 


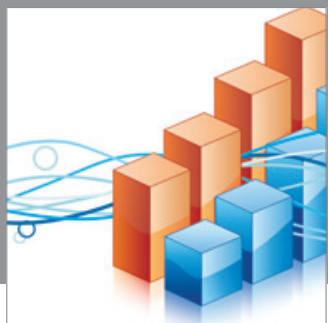

Advances in

Operations Research

mansans

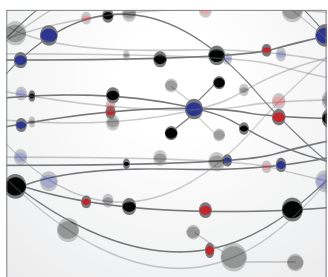

The Scientific World Journal
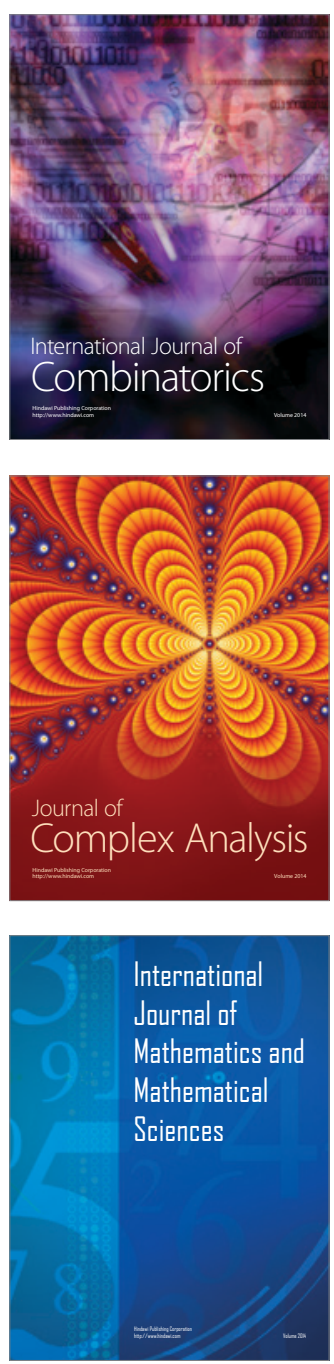
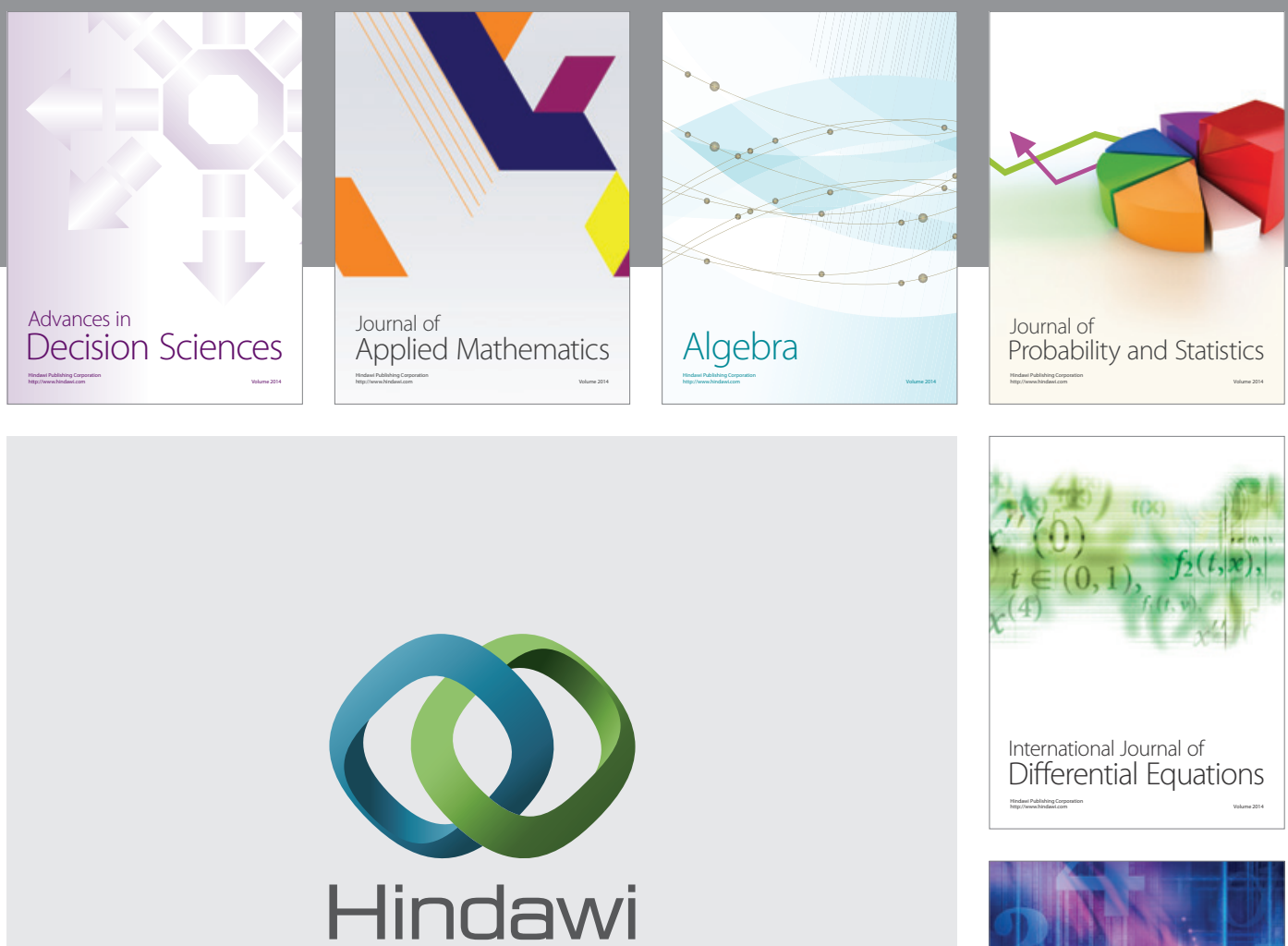

Submit your manuscripts at http://www.hindawi.com
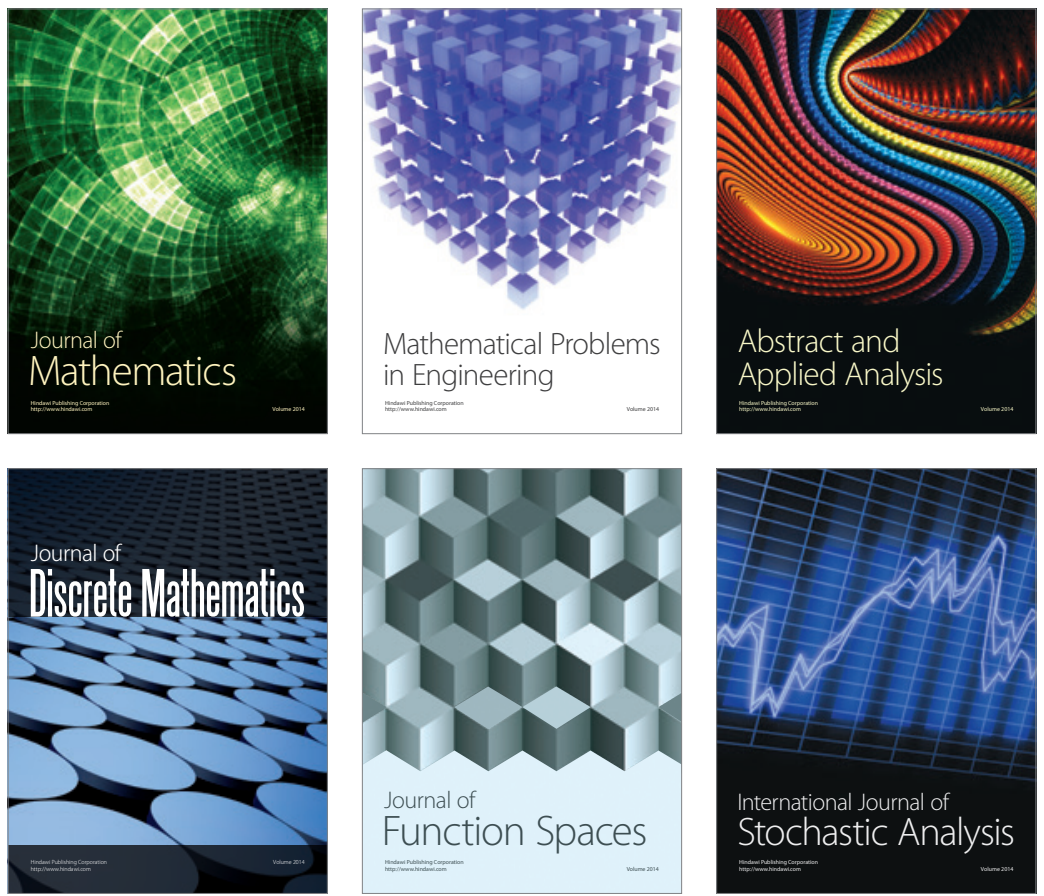

Journal of

Function Spaces

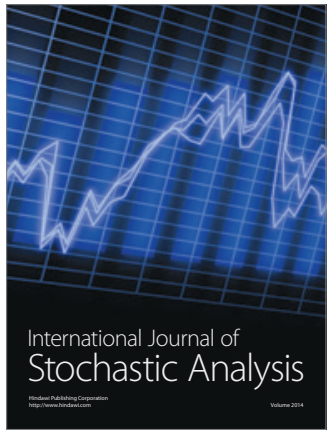

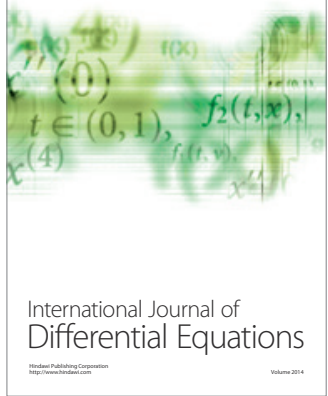
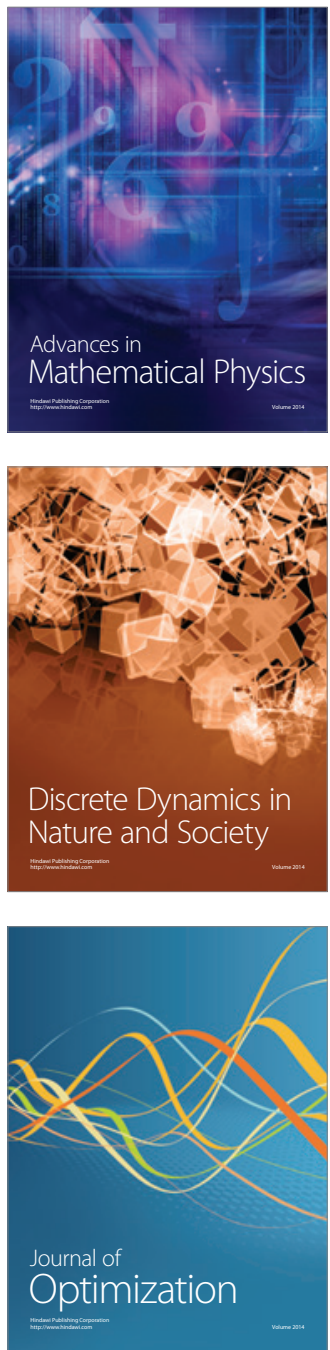